REVESCO. Revista de Estudios Cooperativos

ISSN: $1885-8031$

https://dx.doi.org/10.5209/REVE.67335

\title{
Las medidas fiscales para favorecer el emprendimiento por las cooperativas
}

\author{
Alberto Atxabal Rada ${ }^{1}$ \\ Recibido: 30 de junio de 2019 / Aceptado: 26 de noviembre de 2019 / Publicado: 27 de febrero de 2020
}

Resumen. La normativa tributaria ha adoptado en los últimos años una serie de medidas destinadas a favorecer e impulsar el emprendimiento, que se recogen en las normativas de los principales impuestos directos. Asimismo, entre los emprendedores, y entre los emprendedores sociales debemos situar a las cooperativas, sin duda. En este sentido, las sociedades cooperativas son acreedoras de las medidas fiscales que favorecen el emprendimiento, como cualquier otro emprendedor. Este trabajo se centra en el análisis y crítica de las normas fiscales que promueven el emprendimiento y, a su vez, resultan de aplicación a las cooperativas, o en su caso, no les son aplicables a estas últimas sin que se vislumbre una razón que justifique su omisión.

Estudiaremos, por consiguiente, las medidas fiscales que favorecen el emprendimiento promovido por las cooperativas previstas en la normativa tributaria española, y nos centraremos en el análisis de los principales impuestos directos, es decir, el Impuesto sobre Sociedades y el Impuesto sobre la Renta de las Personas Físicas, por tratarse de los dos impuestos que recogen la mayoría de los incentivos fiscales al emprendimiento en la legislación española. Esta última matización nos obliga a dedicar un apartado propio a las reglas fiscales adoptadas en el País Vasco, que difieren parcialmente de la regulación de territorio común. Así, observamos que el tratamiento fiscal del emprendimiento, cuando lo lleva a cabo una cooperativa, es más generoso en la regulación foral que en la normativa de territorio común, si bien en ambas normativas existen reglas fiscales que impulsan el emprendimiento y que no resultan aplicables a las cooperativas sin una justificación aceptable.

Palabras clave: Tributación; Emprendimiento social; Cooperativas.

Claves Econlit: E62; H20; H24; H30; K34; L31; P13.

\section{[en] Tax measures to encourage entrepreneurship by cooperatives}

Abstract. Tax law has adopted in recent years some of measures to promote and encourage entrepreneurship, which are included in the regulations of the main direct taxes. On the other hand, among entrepreneurs, and among social entrepreneurs, there are cooperatives, no doubt. Therefore, cooperatives have the right to apply tax measures that encourage entrepreneurship, like any other entrepreneur. This work focuses on the analysis and criticism of tax regulations that encourage entrepreneurship and, at the same time, are applicable to cooperatives, or in other case, that are not applicable to cooperatives without any reason for the omission.

We will study, therefore, tax measures that encourage entrepreneurship promoted by the cooperatives, foreseen in Spanish tax regulations, and we will focus on the analysis of the main direct taxes, as Corporation Income Tax and the Personal Income Tax, because both taxes collect most of the tax incentives for entrepreneurship in Spanish legislation. This last circumstance focuses us to remark the tax rules adopted in the Basque Country, which differ partially from the regulation of state laws. Thus, we observe that the tax treatment of entrepreneurship carried out by a cooperative is more generous in Basque regulation than in state regulations, although in both regulations there are fiscal rules to promote entrepreneurship not applicable to cooperatives, without acceptable justification.

Keywords: Taxation; Social entrepreneurship; Cooperatives.

Sumario. 1. Introducción. 2. El concepto de entidad de nueva creación. 3. Los beneficios fiscales de las empresas de nueva creación en el Impuesto sobre Sociedades. 4. La deducción por creación de empleo vinculada al emprendimiento en el Impuesto sobre Sociedades. 5. Medidas fiscales adoptadas en el Impuesto sobre la Renta de las Personas Físicas. 6. Otros incentivos fiscales para el emprendimiento que excluyen a las cooperativas. 7. Conclusiones. 8. Referencias bibliográficas.

Cómo citar: Atxabal Rada, A. (2020) Las medidas fiscales para favorecer el emprendimiento por las cooperativas. REVESCO. Revista de Estudios Cooperativos, vol. 133, e67335. https://dx.doi.org/10.5209/REVE.67335.

\footnotetext{
Universidad de Deusto, España

Dirección de correo electrónico: alberto.atxabal@ deusto.es
} 


\section{Introducción}

No cabe duda de que actualmente se habla mucho de emprendimiento (Varona Alabern, 2018). El emprendimiento supone la acción de iniciar una nueva actividad económica, es decir, estamos hablando de la creación y puesta en marcha de una iniciativa económica, ya sea empresarial o profesional (Global Entrepreunership Monitor, 2018). En sentido estricto, el emprendedor no es todavía empresario, ni su actividad económica constituye una empresa (Belso Martínez et alii, 2014); para ser empresa faltaría consolidar la actividad iniciada, y como veremos en la normativa fiscal que promueve el emprendimiento, se está pensando en un plazo de entre dos y cinco años para lograr dicha consolidación.

El emprendimiento debe ser protegido y fomentado, también por las normas fiscales (Palomo Ruiz, 2015; Luján Alcaraz, 2016; Varona Alabern, 2018). La normativa tributaria ha adoptado en los últimos años una serie de medidas destinadas a favorecer e impulsar el emprendimiento. Desde medidas fiscales tendentes a facilitar la financiación del emprendedor, donde aparecen figuras como los business angels o el crowdfunding, hasta el gravamen de las rentas del emprendedor, sea este persona física o jurídica, todas ellas configuran un conjunto de reglas presididas por una finalidad común, a saber, el impulso del emprendimiento (Ruiz Garijo, 2016).

A la hora de abordar la regulación fiscal del emprendimiento, se hace necesario delimitar el concepto de emprendimiento que vamos a utilizar (Vaquero García, 2016). Y más que hablar de sujetos pasivos emprendedores, es preferible referirnos a la actividad emprendedora, actividad que se puede medir a través de tres indicadores: la producción de patentes de innovaciones, la recepción de fondos de riesgo y la creación de nuevas empresas (Zapata Huamaní, Fernández López y Neira Gómez, 2018), si bien este último indicador es el que mejor reflejaría el emprendimiento. Dejamos a un lado lo que podríamos denominar emprendimiento tecnológico, es decir, aquél que persigue nuevas oportunidades de negocio impulsadas a través de la innovación en ciencia e ingeniería (Zapata Huamaní, Fernández López y Neira Gómez, 2018). Se trataría, por tanto, de proyectos que reúnen medios humanos y materiales, estrechamente relacionados con los avances en el conocimiento científico y tecnológico con el propósito de generar y capturar valor para una empresa. En este caso, no sería necesario crear una nueva empresa para llevar adelante la actividad emprendedora sino que bastaría que una empresa ya existente invirtiera en actividades tecnológicas mediante una rama de actividad o simplemente a través de un departamento de I + D. Sin embargo, en este trabajo no vamos a abordar estas cuestiones y nos centraremos en los incentivos fiscales dirigidos directamente a impulsar la creación y la puesta en marcha de nuevas empresas, en nuestro caso de nuevas empresas cooperativas.

Antes de proceder al análisis pormenorizado de estas reglas fiscales, debemos realizar una última matización debido a la existencia de normativas fiscales forales que difieren de lo regulado en territorio común, por la aplicación de las facultades y potestades contenidas en el Concierto Económico con el País Vasco. Nos vamos a centrar en la regulación de las principales medidas fiscales para el fomento del emprendimiento que han adoptado en los tres Territorios Históricos de Euskadi, siempre que marquen una diferencia con las medidas ya existentes en territorio común. Son dos los impuestos que llaman nuestra atención, a saber, el Impuesto sobre la Renta de las Personas Físicas y el Impuesto sobre Sociedades. Las diferencias entre las regulaciones de estos impuestos en la normativa alavesa, vizcaína y guipuzcoana no son reseñables, por lo que a lo largo de este artículo las referencias se harán a la normativa vizcaína. Todo ello sin perjuicio de que cuando existan diferencias de las normativas alavesa o guipuzcoana con la vizcaína, éstas se podrán de manifiesto.

En cuanto a las diferencias entre la normativa fiscal de territorio común y las propias de los territorios forales, la normativa de los Territorios Históricos de Álava, Bizkaia y Gipuzkoa se asemeja bastante a la normativa estatal por lo que se refiere a la determinación de la base imponible del Impuesto sobre Sociedades. Sin embargo, el tipo de gravamen difiere del tipo de territorio común y, en materia de deducciones, también existen diferencias, sobre todo, cuantitativas. Junto a estas diferencias cuantitativas, que no conceptuales, se recogen otras medidas que no tienen correspondencia o equivalente en la normativa estatal del Impuesto. Así, se abordan medidas que no existen en territorio común como una reserva especial para el fomento del emprendimiento y el reforzamiento de la actividad productiva. Y por otro lado, en Euskadi no se regula un régimen especial para las empresas de reducida dimensión, sino que se han incorporado al régimen general las reglas fiscales previstas para las pequeñas y medianas empresas, si bien se establecen disposiciones específicas para las microempresas y para las pequeñas empresas a lo largo de la regulación general del Impuesto que podría considerarse que conforman tácitamente un régimen fiscal especial (Pérez Delgado, 2014).

Podemos llegar a conclusiones similares respecto al Impuesto sobre la Renta de las Personas Físicas, cuyas normativas difieren en territorio común y en los territorios forales. Estas diferencias se reflejan en la propia estructura del impuesto, en la calificación de algunas rentas, en la aplicación de porcentajes, plazos o cuantías, y en los beneficios fiscales que no coinciden con los regulados en territorio común. Nos 
centraremos en los beneficios fiscales que acogen las reglas fiscales para el impulso del emprendimiento dentro del impuesto, y difieren entre territorio foral y territorio común, como comprobaremos más adelante.

Estas reglas fiscales se las debemos aplicar a los emprendedores, y entre los emprendedores, a las sociedades cooperativas (Chaves y Monzón, 2018). La cooperativa se muestra como un medio adecuado para materializar el emprendimiento, y más concretamente, el emprendimiento social. Por otra parte, la normativa tributaria ha adoptado en los últimos años una serie de medidas destinadas a favorecer e impulsar el emprendimiento. La fiscalidad constituye un instrumento idóneo para impulsar el emprendimiento, estableciendo incentivos fiscales que incentiven el inicio de nuevas actividades económicas y negocios profesionales (Varona Alabern, 2018). Desde medidas fiscales tendentes a facilitar la financiación del emprendedor, donde aparecen figuras como los business angels o el crowdfunding, hasta el gravamen de las rentas del emprendedor, sea este persona física o jurídica, todas ellas configuran un conjunto de reglas presididas por una finalidad común, a saber, el impulso del emprendimiento. La cooperativa opera, pues, como un catalizador en el que confluyen los dos ejes anteriores (Arnáez Arce, 2018b). Este trabajo se centra en el análisis y crítica de las normas fiscales que promueven el emprendimiento y, a su vez, resultan de aplicación a las cooperativas, o en su caso, no les son aplicables a estas últimas sin que se vislumbre una razón que justifique su omisión. Estudiaremos, por consiguiente, las medidas fiscales que favorecen el emprendimiento promovido por las cooperativas, previstas en la normativa tributaria española.

La normativa tributaria del Impuesto sobre Sociedades y del Impuesto sobre la Renta es más bien parca a la hora de plasmar incentivos fiscales dirigidos al emprendimiento, y las referencias se centran en las entidades de nueva creación, si bien son relativamente escasas. Por ejemplo, en el Impuesto sobre Sociedades, recordemos que se refieren a ellas directamente cuando regula el tipo de gravamen, la compensación de bases imponibles negativas, y la deducción por creación de empleo.

Dividiremos el artículo en dos grandes bloques que reflejarán las medidas adoptadas en la imposición sobre la renta de las personas físicas y de las personas jurídicas.

Por una parte, se analizarán las medidas fiscales recogidas en la normativa del Impuesto sobre Sociedades que afectan o se refieren a las empresas de nueva creación, partiendo del concepto tributario que utiliza el artículo 29.1 de la Ley 27/2014, de 27 de noviembre, del Impuesto sobre Sociedades ${ }^{2}$ (en adelante LIS). Analizaremos, por tanto, la compensación de bases imponibles negativas cuando la base pertenece a una empresa de nueva creación, el tipo de gravamen de las mismas, o las reglas especiales a la hora de aplicar las deducciones por inversiones. Son aspectos de la liquidación del Impuesto donde se establecen reglas especiales, generalmente menos onerosas, para las empresas de nueva creación.

Y por otra parte, sumaremos el estudio de la deducción por creación de empleo a través del contrato de apoyo a los emprendedores que no está relacionado directamente con las entidades de nueva creación, sino que resulta aplicable a cualquier clase de empresa sometida al Impuesto sobre Sociedades.

En segundo lugar, estudiaremos las reglas fiscales previstas en la normativa del IRPF ${ }^{3}$ para impulsar el emprendimiento, y que, a su vez, resulten de aplicación a las cooperativas o a los socios de las mismas.

Comenzaremos, por tanto, deslindando el concepto de entidad de nueva creación, que vertebra buena parte de las medidas fiscales para el emprendimiento que recoge el Impuesto sobre Sociedades, y que difiere del concepto que utiliza el Impuesto sobre la Renta de las Personas Físicas, como veremos.

\section{El concepto de entidad de nueva creación}

La LIS, en su artículo 29, no define qué debemos entender por entidad o empresa de nueva creación, si bien es un concepto que utiliza la normativa del Impuesto en diversas ocasiones. Se trata, en todo caso, de un concepto universal, aplicable a todo tipo de empresa, si bien en nuestro caso, debemos analizarlo desde el punto de vista de las cooperativas. Por tanto, cualquier clase de empresa puede ser una entidad de nueva creación, y entre ellas, la cooperativa. Cuando la cooperativa sea una entidad de nueva creación, deberá poder aplicar los incentivos fiscales al emprendimiento a semejanza de lo que ocurriría con otras clases de empresa.

El concepto de entidad de nueva creación en el Impuesto sobre Sociedades y en el IRPF no coinciden exactamente. Se aleja en cierta medida la regulación del Impuesto sobre Sociedades de la prevista en la normativa del Impuesto sobre la Renta de las Personas Físicas a partir de la redacción dada por el Real Decreto-Ley 4/2013, de 22 de febrero, de medidas de apoyo al emprendedor y de estímulo del crecimiento y

Publicada en el Boletín Oficial del Estado, núm. 288, de 28 de noviembre de 2014.

Cfr. Ley 35/2006, de 28 de noviembre, del Impuesto sobre la Renta de las Personas Físicas y de modificación parcial de las leyes de los Impuestos sobre Sociedades, sobre la Renta de no Residentes y sobre el Patrimonio (Boletín Oficial del Estado, núm. 285 , de 29 de noviembre de 2006). 
de la creación de empleo ${ }^{4}$. Por su parte, la Ley 14/2013, de 27 de septiembre, de apoyo a los emprendedores y su internacionalización ${ }^{5}$, en su artículo 27. Cuatro fijó en la normativa del IRPF, en su artículo 68.1 de entonces, que permanece en el artículo actual, las características que debía cumplir una entidad de nueva creación para ser acreedora de los beneficios fiscales previstos en el IRPF.

La normativa del IRPF exige que la entidad de nueva creación tenga las siguientes características:

a) Revestir la forma de sociedad anónima, sociedad de responsabilidad limitada, sociedad anónima laboral, sociedad de responsabilidad limitada laboral. Curiosamente, la norma del IRPF se olvida de las cooperativas, a diferencia de la norma del Impuesto sobre Sociedades que sí las contempla.

b) No estar admitida a negociación en ningún mercado organizado.

c) Ejercer una actividad económica que cuente con los medios personales y materiales para el desarrollo de la misma.

d) El importe de la cifra de los fondos propios de la entidad no puede ser superior a 400.000 euros en el inicio del periodo impositivo en que el contribuyente adquiera sus acciones o participaciones.

Asimismo, para gozar de la deducción la inversión se debe producir en el momento de la constitución o mediante la ampliación de capital efectuada en los tres años siguientes a dicha constitución.

Por el contrario, para que una nueva empresa sea considerada una entidad de nueva creación en el Impuesto sobre Sociedades deben cumplir una serie de requisitos que no coinciden plenamente con los que acabamos de mencionar en la normativa del IRPF (Atxabal Rada, 2018b). Los requisitos son, a saber:

a) Es necesario que la entidad se haya constituido a partir del 1 de enero de $2015^{6}$.

b) La entidad deberá iniciar el desarrollo de alguna actividad económica, es decir, deberá ordenar por cuenta propia los medios de producción y los recursos humanos o uno de los dos con la finalidad de intervenir en la producción o distribución de bienes y servicios en el mercado.

Como observamos, este concepto de entidad de nueva creación difiere del utilizado en la normativa del IRPF, por lo que a la deducción por inversión en este tipo de entidades se refiere (Atxabal Rada, 2018b). Así, en el IRPF no se admitiría la deducción para la inversión en una cooperativa, aunque sí sería posible invertir en una sociedad laboral; mientras que en el Impuesto sobre Sociedades el concepto es mucho más amplio acogiendo también a las cooperativas, cuya regulación fiscal específica (Ley 20/1990) recoge expresamente la aplicación de la reducción del tipo de gravamen a las cooperativas que sean entidades de nueva creación. Lo cierto es que el Impuesto sobre Sociedades es más consecuente con el concepto que pretende regular por lo que solo exige que sea nueva la actividad y la entidad que lleva a cabo la misma, sin preocuparle la forma jurídica que adopte la entidad. Sin duda, es una regulación mucho más neutra que la del IRPF.

Por otro lado, a la vista de estos dos requisitos, no se exige una cifra mínima de negocios para acogerse al tipo de gravamen reducido, ni un número mínimo de empleados, por lo que cualquier contribuyente del Impuesto sobre Sociedades de nueva constitución que cumpla los citados requisitos podrá beneficiarse del tipo de gravamen del $15 \%$ durante dos años.

\subsection{Exclusión de la calificación como entidades de nueva creación}

Además de su creación a partir de 2015 y el inicio de una nueva actividad económica, circunstancias necesarias para considerar que nos encontramos ante entidades de nueva creación a efectos del Impuesto sobre Sociedades, el artículo 29.1 de la LIS establece algunos supuestos de exclusión de nuevas entidades que se creen, bien porque entiende que no inician una nueva actividad, bien porque no realizan actividad económica alguna, bien porque pertenecen a un grupo mercantil y, aunque inicien una nueva actividad, la ley no quiere premiar el emprendimiento que llevan a cabo los grupos de empresa (Romero Flor y Álamo Cerrillo, 2014b). La norma gira en torno al concepto de entidad de nueva creación y no en torno al concepto de inicio de la actividad. Estas causas de exclusión no van a resultar aplicables a las cooperativas con carácter general porque regulan situaciones propias de las sociedades de capital que no suelen tener reflejo en el ámbito cooperativo.

\footnotetext{
4 Publicado en el Boletín Oficial de Estado, núm. 47, de 23 de febrero de 2013. Este Real Decreto-Ley introdujo una serie de beneficios fiscales en la normativa del Impuesto sobre Sociedades y del IRPF, con efectos desde el 1 de enero de 2013, para las entidades de nueva creación y para los socios de las mismas.

Publicada en el Boletín Oficial de Estado, núm. 233, de 28 de septiembre de 2013.

6 Las sociedades de capital se constituyen mediante escritura pública e inscripción en el Registro Mercantil, teniendo la inscripción carácter constitutivo, por tanto, la fecha que debemos tomar en consideración para constatar la constitución de la sociedad de cara a la aplicación del límite temporal del 1 de enero de 2015 es la fecha en que la sociedad se inscriba en el Registro Mercantil. CV1210-15, de 17 de abril de 2015. En virtud de la Disposición Transitoria vigésima segunda de la LIS, estos incentivos para las entidades de nueva creación van a ser de aplicación incluso a las entidades de nueva creación constituidas entre el 1 de enero de 2013 y el 31 de diciembre de 2014 , respecto de los periodos impositivos iniciados a partir del 1 de enero de 2015 en los que se cumplan las condiciones establecidas.
} 


\subsubsection{Actividad transmitida por una persona vinculada}

A estos efectos, no se entenderá iniciada una actividad económica cuando la actividad económica hubiera sido realizada con carácter previo por otras personas o entidades vinculadas en el sentido del artículo 18 de la LIS y transmitida, por cualquier título jurídico, a la entidad de nueva creación. Se trata de un supuesto de difícil traslación al ámbito cooperativo, salvo para supuestos de integración cooperativa de segundo o ulterior grado.

\subsubsection{Actividad transmitida por una persona física que controla el capital de la entidad de nueva creación}

Tampoco se entiende iniciada una actividad económica cuando la actividad económica hubiera sido ejercida, durante el año anterior a la constitución de la entidad, por una persona física que ostente una participación, directa o indirecta, en el capital o en los fondos propios de la entidad de nueva creación superior al $50 \%$. En el caso de las cooperativas de primer grado es difícil que se pueda producir esta circunstancia porque las leyes de cooperativas, tanto la estatal como las autonómicas, limitan el porcentaje del capital social que pueda acumular un socio de la cooperativa a un tercio (art. 45.6 de la Ley estatal y 53.4 de la Ley vasca, si bien en este último caso se admiten participaciones mayores en el capital para el supuesto de que la cooperativa tenga menos de diez socios). Asimismo, tampoco será sencillo que esta situación se produzca en las cooperativas de segundo grado, puesto que se exige la participación en el capital de otras cooperativas además de la persona física que transmite la actividad.

No se podrá aplicar el tipo reducido, sin embargo, en los casos de transformación social. Cuando una sociedad se transforma en otra clase de sociedad pero continúa realizando la misma actividad. Por ejemplo, en el caso de una sociedad civil sin personalidad jurídica que tributaba en el régimen de atribución de rentas a sus socios que se transforma en una sociedad limitada no es posible la aplicación del tipo reducido, porque la actividad la desarrollaban los socios a efectos fiscales, y son éstos los que la aportan a la sociedad limitada (CV2170-16, de 19 de mayo de 2016). Podríamos extrapolar estas conclusiones a la transformación en sociedad cooperativa de sociedades civiles o de comunidades de bienes.

\subsubsection{Entidad de nueva creación perteneciente a un grupo mercantil}

Afirma el artículo 29.1 de la LIS, que no tendrán la consideración de entidades de nueva creación aquellas que formen parte de un grupo en los términos establecidos en el artículo 42 del Código de Comercio, con independencia de la residencia y de la obligación de formular cuentas anuales consolidadas. Esta posibilidad se debería adaptar al ámbito cooperativo donde las fórmulas de integración societaria parten de la creación de cooperativas de segundo y ulterior grado a partir de sociedades cooperativas de primer grado que son las que controlan a la cooperativa de segundo grado. Por consiguiente, la exclusión fiscal se dirige a las cooperativas de segundo o ulterior grado que sean, a su vez, entidades de nueva creación, las cuales no podrían acogerse a los incentivos al emprendimiento, si las cooperativas de primer grado ostentan una toma de control sobre las cooperativas de segundo o ulterior grado, aunque desde una perspectiva de Derecho mercantil no puedan conformar un grupo mercantil por carecer las cooperativas de dicha naturaleza.

\subsubsection{Entidad de nueva creación considerada patrimonial}

El tipo de gravamen del 15 por ciento previsto en este apartado no resultará de aplicación a aquellas entidades que tengan la consideración de entidad patrimonial, en los términos establecidos en el apartado 2 del artículo 5 de la LIS. Esta última causa es de escasa aplicabilidad en el caso de las cooperativas.

\section{Los beneficios fiscales de las empresas de nueva creación en el Impuesto sobre Sociedades}

En lo que respecta a las medidas aplicables a las entidades de nueva creación previstas en el Impuesto sobre Sociedades hay que señalar que las mismas se centran principalmente en una disminución del tipo impositivo, una compensación sin limitación de bases imponibles negativas y la aplicación de varias deducciones. Estos incentivos fiscales podrían resultar positivos para las entidades de nueva creación, ya que se traducen en una menor carga fiscal para los sujetos pasivos (Romero Flor y Álamo Cerrillo, 2014a).

Antes de analizar la fiscalidad de las entidades de nueva creación, debemos exponer someramente la tributación de las cooperativas. A la sociedad cooperativa se le aplican las reglas fiscales propias de cualquier clase de sociedad de capital o mercantil, con algunas especialidades. La especificidad de la fiscalidad de las cooperativas responde a dos razones (Alonso Rodrigo, 2001): por un lado, se fijan una serie de reglas que pretenden la adecuación de las normas tributarias a las características de una sociedad 
cooperativa que le diferencian de las sociedades capitalistas y que, por este motivo, estas reglas fiscales especiales resultan de aplicación a todas las sociedades cooperativas. Son las que la doctrina denomina reglas de ajuste. Y, por otro lado, se establecen una serie de beneficios fiscales para aquellas sociedades cooperativas que cumplan determinados requisitos, estableciendo una clasificación de las cooperativas a efectos fiscales que tiene su reflejo en la protección que les otorga el ordenamiento jurídico-tributario. Así hablamos de cooperativas protegidas o especialmente protegidas junto a cooperativas no protegidas, calificaciones todas ellas que se limitan al ámbito tributario sin que tengan incidencia o reflejo alguno fuera del mismo, en el ámbito civil, mercantil o laboral. Esta fiscalidad específica para las cooperativas responde, a su vez, a sus reglas especiales de funcionamiento.

La característica más singular de su régimen fiscal es la división de la base imponible en dos clases de rentas: las que derivan de los resultados cooperativos, esto es, de las relaciones entre las cooperativas y sus socios; y las demás rentas que se denominan resultados extracooperativos (Montero Simó, 2016). Esta clasificación de los resultados tiene su incidencia en la fijación de la cuota a pagar. Así, en el caso de las cooperativas fiscalmente protegidas o especialmente protegidas a las que hemos hecho referencia, el tipo de gravamen aplicable a los resultados cooperativos va a ser inferior al tipo general del Impuesto, siendo éste en la actualidad el mayor beneficio fiscal que recoge su régimen fiscal especial.

\subsection{La compensación de bases imponibles negativas}

Como sabemos, en virtud del artículo 26 de la LIS, actualmente se permite la compensación de bases imponibles negativas producidas en ejercicios anteriores sin limitación en cuanto al plazo para compensar esas bases negativas, pero limitadas a una determinada cuantía (Atxabal Rada, 2014). Así, nos dice el artículo 26, en su apartado primero, que las bases imponibles negativas que hayan sido objeto de liquidación o autoliquidación podrán ser compensadas con las rentas positivas de los períodos impositivos siguientes con el límite del 70\% de la base imponible previa a la aplicación de la reserva de capitalización establecida en el artículo 25 de la LIS y previa también a su compensación de la base imponible generada en el periodo impositivo en que se pretende aplicar esta opción del Impuesto.

La única salvedad a esta limitación cuantitativa es que, en todo caso, se podrán compensar en el período impositivo bases imponibles negativas hasta el importe de 1 millón de euros.

Una vez comentado a grandes rasgos el régimen de compensación de bases imponibles negativas, por lo que a nosotros nos interesa, debemos mencionar una regla especial aplicable a las entidades de nueva creación en este ámbito. El mismo artículo 26 de la LIS, en su apartado tercero, establece que el límite del $70 \%$ no resultará de aplicación en el caso de entidades de nueva creación que cumplan las características para poder aplicar el tipo de gravamen especial del 15\% previsto en el artículo 29.1 de la LIS, en los 3 primeros períodos impositivos en que se genere una base imponible positiva previa a su compensación. Así, las entidades de nueva creación pueden compensar la totalidad de sus bases imponibles negativas sin limitación alguna en los tres primeros periodos impositivos en los que la base imponible sea positiva. Este incentivo se dirige a emprendedores societarios que ya se encuentran en fase de crecimiento y consolidación, pero deja fuera a los nacientes (Lagos Rodríguez y Álamo Cerrillo, 2016).

Sin embargo, en virtud del artículo 24 de la Ley 20/1990, de 19 de diciembre, sobre Régimen Fiscal de las Cooperativas ${ }^{7}$, en las cooperativas a las que resulte de aplicación la normativa fiscal de régimen común, la compensación no se realiza entre las bases, sino que se hace entre las cuotas. Así, si la suma algebraica de las cantidades resultantes de aplicar los tipos de gravamen correspondientes a las bases imponibles derivadas de los resultados cooperativos y extracooperativos, positivas o negativas, resultase negativa, su importe podrá compensarse por la cooperativa con las cuotas íntegras positivas de los periodos impositivos siguientes, con el límite del 70 por ciento de la cuota íntegra previa a su compensación (Montero Simó, 2016). En todo caso, serán compensables en el período impositivo cuotas íntegras por el importe que resulte de multiplicar un millón de euros al tipo medio de gravamen de la entidad. Este procedimiento sustituye a la compensación de bases imponibles negativas prevista en el artículo 26 de la LIS que, en consecuencia, no será aplicable a las cooperativas.

Lamentablemente, no hay ninguna mención a las cooperativas que, a su vez, sean entidades de nueva creación. Asimismo, el artículo 24 de la Ley 20/1990 excluye expresamente la aplicación del artículo 26 de la LIS, donde se recoge la regla especial para las entidades de nueva creación, por lo que cierra cualquier opción de aplicación analógica del mismo a las cooperativas que sean entidades en nueva creación. La ley especial, en este caso la Ley 20/1990, prevalece sobre la ley general, la LIS. En definitiva, las cooperativas que, a su vez, sean entidades de nueva creación no podrán acogerse a la regla más favorable que les permitiría deducirse las bases imponibles negativas en los tres primeros periodos en que generen bases 
imponibles positivas, sin limitación alguna. Podemos concluir, por consiguiente, que las cooperativas sufren un peor tratamiento fiscal que otros contribuyentes del Impuesto.

En Euskadi, la regulación fiscal difiere de la de territorio común. La mayoría de las rentas de la cooperativa van a la base general por lo que no se diferencia entre resultados cooperativos y extracooperativos. A lo sumo, puede haber rentas que conformen la base imponible especial ${ }^{8}$, pero no hay diferencia entre las rentas generadas por la cooperativa por lo que el sumatorio de todas ellas conforman la base imponible. En cuanto a la compensación de bases imponibles negativas correspondientes a periodos anteriores, se podrán compensar por un plazo de treinta años con el límite del 50\% de la base imponible positiva previa a dicha compensación, o del $70 \%$ para las microempresas y pequeñas empresas, salvo que nos encontremos en procedimientos concursales donde no se aplicará limitación alguna (Armentia Basterra, 2014).

Al igual que sucede en territorio común, en Euskadi, las entidades de nueva creación podrán computar el plazo de compensación a partir del primer período impositivo cuya base imponible sea positiva, si bien tendrá que aplicar los límites cuantitativos que le correspondan en función de su tamaño, por lo que su tratamiento fiscal es más gravoso que en territorio común donde el límite no se aplica en los tres primeros ejercicios con rentas positivas (Arrázola Arrién, 2015). No existe, sin embargo, discriminación alguna para las cooperativas, puesto que esta regla más perjudicial que la de territorio común se aplica a cualquier clase de empresa que sea sujeto pasivo del Impuesto sobre Sociedades, es decir, que se aplica por igual a todas las empresas, sean cooperativas o no lo sean.

\subsection{El tipo de gravamen}

Ya nos advierte la Exposición de Motivos de la LIS que, en el caso de entidades de nueva creación, el tipo de gravamen se mantiene en el 15\% para el primer período impositivo en que obtienen una base imponible positiva y el siguiente. Y además, no resulta aplicable este tipo del $15 \%$ si la entidad de nueva creación debiera tributar por un tipo inferior como pudiera ser un $10 \%$, un $1 \%$ o un $0 \%$, por tratarse de una entidad sin fines lucrativos, una sociedad o un fondo de inversión, un fondo de activos bancarios o un fondo de pensiones (Montesinos Oltra, 2017).

Las entidades de nueva creación, que a su vez sean cooperativas, se mencionan en relación a la aplicación del tipo de gravamen sobre las distintas clases de resultados que conforman su base imponible. Así en el caso de las cooperativas fiscalmente protegidas, a la base imponible correspondiente a los resultados cooperativos se les aplica un tipo del $20 \%$ mientras que a los resultados extracooperativos se les aplica el tipo general del Impuesto. Sin embargo, si la cooperativa cumple los requisitos para ser considerada entidad de nueva creación podrá aplicar el $15 \%$ tanto sobre los resultados cooperativos como sobre los resultados extracooperativos ${ }^{9}$ en el primer periodo impositivo que obtengan resultados positivos y el siguiente inmediato, al desaparecer toda referencia al tipo general del impuesto que resultase aplicable sobre los resultados extracooperativos.

En el caso de las cooperativas especialmente protegidas se establece una regla similar (Montero Simó, 2016). Estas cooperativas tienen derecho a aplicar una bonificación del $50 \%$ de la cuota íntegra del Impuesto, obtenida mediante la suma de las dos cuotas derivadas de la aplicación de los tipos de gravamen que utilizan las cooperativas protegidas sobre los resultados cooperativos (el 20\%) y extracooperativos (el tipo general del Impuesto). Pues bien, si la cooperativa especialmente protegida se puede calificar de entidad de nueva creación, podrán aplicar su bonificación del 50\% de la cuota íntegra que se deriva de la aplicación del $15 \%$ sobre los resultados tanto cooperativos como extracooperativos. De esta forma, el tipo efectivo de gravamen durante el primer periodo impositivo en que obtengan rentas positivas y el inmediato siguiente será del 7,5\% sobre todos los resultados que integran la base imponible.

La aplicación de este tipo reducido, sin embargo, tiene una limitación temporal tal y como hemos visto. El $15 \%$ es aplicable únicamente en el primer periodo impositivo desde su constitución en el que la entidad de nueva creación obtenga rentas positivas, así como en el periodo impositivo siguiente. De la dicción literal del artículo parece deducirse que no es necesario tener resultado positivo en el segundo periodo. Si en el periodo impositivo inmediatamente posterior al primero en que la entidad ha obtenido una base imponible positiva dicha entidad obtiene una renta negativa, no parece que pueda aplazarse la aplicación del tipo del 15\% hasta un periodo posterior en que obtenga una renta positiva, por lo que la efectividad de este incentivo queda un tanto en entredicho. Asimismo, no importa que la entidad haya actuado todo el ejercicio o una parte del mismo para la aplicación de la regla (Memento Fiscal, 2018). Es decir, que si una sociedad se constituye a

\footnotetext{
8 Los rendimientos íntegros de capital mobiliario, con excepción de los retornos cooperativos, de los rendimientos obtenidos por la cesión a terceros de capitales propios que, de acuerdo con lo dispuesto en los correspondientes convenios para evitar la doble imposición, no estén sometidos a retención o ingreso a cuenta y de los dividendos que den derecho a la aplicación de la no integración del 100\% en la base imponible del Impuesto sobre Sociedades.

9 Para que sea así, fueron necesarias dos modificaciones de la normativa del Impuesto, lo que sin duda debe atribuirse a un lapsus del legislador (Montesinos Oltra, 2017).
} 
mediados del ejercicio pero al final del mismo ha generado alguna base imponible positiva, ya se habría cumplido el requisito de ser el primer periodo con renta positiva y debería tributar al $15 \%$.

A diferencia de la normativa de territorio común, la normativa foral del Impuesto no regula a las entidades de nueva creación ni les establece un tipo de gravamen diferenciado durante los dos primeros ejercicios con resultado positivo de la base imponible. En su lugar, se establece un tipo general del $24 \%$, de aplicación a partir del 1 de enero de 2019, y un tipo reducido para las microempresas y las pequeñas empresas del 20\%, con independencia de que estén o no iniciando su actividad. Ahora bien, las microempresas pueden acabar aplicando un tipo efectivo menor si optan por minorar el $10 \%$ de su base imponible positiva sin necesidad de justificar esa reducción, quedando su tipo efectivo en el 18\% (Armentia Basterra, 2014). Las cooperativas, por su parte, tributan al tipo general si no están protegidas fiscalmente. Las cooperativas fiscalmente protegidas tributan al $20 \%$, salvo que se consideren microempresas o pequeñas empresas en cuyo caso tributan al $18 \%$. Y las cooperativas especialmente protegidas pueden aplicar una bonificación del $50 \%$ sobre la cuota previamente fijada conforme a los tipos citados.

\subsection{Normas comunes a las deducciones}

Junto a las reglas anteriores, y por lo que se refiere a las normas comunes aplicables a las deducciones por inversiones, el artículo 39 de la LIS establece unas medidas para la correcta aplicación de las deducciones por inversiones siguiendo un orden prefijado y regulando aquellas situaciones en que el importe de las deducciones supera un límite de la cuota íntegra ajustada positiva y, por tanto, no se puede minorar el importe total de la deducción por insuficiencia de cuota. En estos casos, se traslada el derecho a la deducción del exceso no minorado a periodos posteriores mediante la creación de un crédito fiscal frente a la Hacienda pública. En esta tesitura, el artículo 39 de la LIS se acuerda de las entidades de nueva creación fijando una medida más favorable a los intereses de la entidad.

Así, el artículo 39 permite las deducciones por inversiones una vez practicadas, en su caso, las deducciones por doble imposición y las bonificaciones del Impuesto, es decir, una vez obtenida la cuota íntegra ajustada con saldo positivo. No obstante, no existe una minoración ilimitada de las deducciones por inversiones, sino que se regula un límite conjunto de todas ellas por lo que no pueden superar el $25 \%$ de la cuota íntegra ajustada positiva. El exceso no minorado se podrá compensar en años posteriores.

Las cantidades no deducidas, por tanto, podrán aplicarse en las liquidaciones de los períodos impositivos que concluyan en los 15 años inmediatos y sucesivos. No obstante, las cantidades correspondientes a la deducción por investigación y desarrollo e innovación tecnológica podrán aplicarse en las liquidaciones de los períodos impositivos que concluyan en los 18 años inmediatos y sucesivos. Si durante estos plazos tampoco existe una cuota suficiente para efectuar la deducción, se pierde el derecho a la misma.

El cómputo de los plazos para la aplicación de las deducciones por inversiones podrá diferirse hasta el primer ejercicio en que, dentro del período de prescripción, se produzcan resultados positivos, en el supuesto de las entidades de nueva creación, entre otras. El inicio del cómputo de los 15 ó 18 años se retrasa, por consiguiente, hasta que la entidad de nueva creación obtenga rentas positivas. En estos casos pueden quedar saldos pendientes de aplicar más allá del transcurso de los años previstos con carácter general para la aplicación de la deducción pero no más allá de cuatro años que coincide con el plazo de prescripción del Impuesto.

Por otra parte, en Euskadi, el límite previsto para la suma de deducciones por inversiones distintas de las correspondientes a investigación y desarrollo e innovación tecnológica ha pasado del $45 \%$ al 35\% de la cuota líquida. Por otra parte, se establece un límite para las deducciones por actividades de investigación y desarrollo e innovación tecnológica, que se fija en el $70 \%$ sobre el exceso de cuota líquida que resulte una vez aplicado el límite conjunto de deducciones del 35\%, que con anterioridad no estaban sometidas a límite cuantitativo que no fuera el importe de la propia cuota líquida en su integridad. Además, se amplía de quince a treinta el número de períodos impositivos en los que se podrán deducir las cantidades no deducidas por insuficiencia de cuota íntegra. Sin embargo, no hay ninguna regla específica para las entidades de nueva creación ni para las cooperativas, que deberán, en todo caso, aplicar el régimen general.

\section{La deducción por creación de empleo vinculada al emprendimiento en el Impuesto sobre Sociedades}

Además de las reglas que acabamos de analizar y que se dirigen fundamentalmente al establecimiento de incentivos fiscales para las entidades de nueva creación, la normativa del Impuesto sobre Sociedades contiene otros beneficios fiscales que favorecen la creación de empleo relacionado con el emprendimiento.

El artículo 37 de la LIS establece dos deducciones por creación de empleo que están directamente vinculadas al emprendimiento. Se trata de deducciones por creación de empleo como consecuencia de la 
firma de contratos de trabajo de apoyo a los emprendedores, regulados en la Ley 3/2012, de 6 de julio, de medidas urgentes para la reforma del mercado laboral ${ }^{10}$. Este contrato de trabajo debe firmarse por tiempo indefinido por escrito, y solo puede concertarse por empresas que tengan menos de cincuenta trabajadores en el momento de producirse la contratación.

Como decíamos, la empresa que celebre este tipo de contrato tendrá derecho a aplicar dos deducciones. La primera se refiere a las entidades que contraten a su primer trabajador a través de un contrato de trabajo por tiempo indefinido de apoyo a los emprendedores, que sea menor de 30 años. En este caso, la entidad podrá deducir de la cuota íntegra del Impuesto la cantidad de 3.000 euros. Se trata, por tanto, de empresas que no tienen trabajadores con carácter previo a la nueva contratación, bajo la modalidad de contrato de trabajo de apoyo a los emprendedores.

En segundo lugar, además de la deducción ya comentada en el párrafo anterior, las entidades que tengan una plantilla inferior a 50 trabajadores en el momento en que concierten contratos de trabajo por tiempo indefinido de apoyo a los emprendedores, con desempleados beneficiarios de una prestación contributiva por desempleo de la Seguridad Social, podrán deducir de la cuota íntegra el 50\% del menor de los siguientes importes:

a) El importe de la prestación por desempleo que el trabajador tuviera pendiente de percibir en el momento de la contratación.

b) El importe correspondiente a doce mensualidades de la prestación por desempleo que tuviera reconocida.

Por su parte, en Euskadi, y por lo que se refiere a la deducción por creación de empleo no hay ninguna referencia al contrato de trabajo de apoyo a los emprendedores. En su lugar, y con el objeto favorecer la creación de puestos de trabajo de calidad y debidamente remunerados, se mejora la regulación de la deducción por creación de empleo, de tal manera que, para aplicar la deducción, se exige que el salario de la persona contratada de manera indefinida sea superior al $170 \%$ del salario mínimo interprofesional vigente en el momento de la contratación. Por otra parte, la cuantía de la deducción se vincula con el salario de la persona trabajadora, siendo con carácter general del 25\% del salario anual bruto con un límite de 5.000 euros. Cuando la persona contratada se encuentre incluida en alguno de los colectivos de especial dificultad de inserción en el mercado de trabajo, la deducción determinada de conformidad con las reglas anteriores se duplica.

En el caso de las cooperativas, y a la luz del artículo 26 de la Ley 20/1990 y el homónimo artículo 28 de la normativa fiscal especial de cooperativas en Bizkaia, la deducción por creación de empleo prevista en el Impuesto sobre Sociedades será de aplicación, además de en los supuestos y con los requisitos establecidos para cada ejercicio económico, a la admisión definitiva, una vez superado el período de prueba, de nuevos socios en las cooperativas de trabajo asociado o, en general, de socios de trabajo en cualquier cooperativa. Habrá que entender, por tanto, que la admisión definitiva del socio trabajador o del socio de trabajo permite la aplicación de la deducción por la creación de empleo prevista en el artículo 37 de la LIS, que hemos explicado.

\section{Medidas fiscales adoptadas en el Impuesto sobre la Renta de las Personas Físicas}

El IRPF también regula incentivos que impulsan el emprendimiento y que, al mismo tiempo, son aplicables a las cooperativas, si bien alguno de ellos no lo pueden utilizar las cooperativas en todo el país. Así, se prevé una exención por el cobro de las prestaciones por desempleo, y dos deducciones, una por la inversión en entidades de nueva o reciente creación y otra por inversión en fondos europeos para el impulso de la innovación.

\subsection{La exención por el cobro en forma de capital de las prestaciones por desempleo}

Dispone el apartado n) del artículo 7 de la Ley del IRPF que están exentas las prestaciones por desempleo reconocidas por la respectiva entidad gestora cuando se perciban en la modalidad de pago único establecida en el Real Decreto 1044/1985, de 19 de junio, por el que se regula el abono de la prestación por desempleo en su modalidad de pago único, siempre que las cantidades percibidas se destinen a las finalidades y en los casos previstos en la citada norma. En concreto, establece el artículo 1 de dicho Real Decreto que los titulares de la prestación por desempleo, del nivel contributivo, puedan percibir de una sola vez la prestación siempre que acrediten ante el INEM que van a realizar una actividad profesional como trabajadores autónomos o socios de una cooperativa de trabajo asociado o sociedad que tenga el carácter de laboral 
(Atxabal Rada, 2018a). Se permite, por tanto y por lo que aquí nos interesa, la percepción de la prestación para convertirse el trabajador desempleado en un trabajador estable en una cooperativa existente o de nueva creación (Arranz de Andrés, 2018).

Esta exención estará condicionada al mantenimiento de la acción o participación durante el plazo de cinco años, en el supuesto de que el contribuyente se hubiera integrado en sociedades laborales o cooperativas de trabajo asociado o hubiera realizado una aportación al capital social de una entidad mercantil, o al mantenimiento, durante idéntico plazo, de la actividad, en el caso del trabajador autónomo. Se refuerza así el incentivo al autoempleo a través, entre otras posibilidades, de la incorporación del trabajador desempleado a cooperativas de trabajo asociado (Montesinos Oltra, 2017).

En todo caso, puede resultar no poco frecuente en la práctica que el negocio o la actividad emprendida resulte finalmente infructuoso, trayendo consigo el cese de la actividad, por causas ajenas a la voluntad del emprendedor. El legislador debería haber tenido en cuenta esta posibilidad (Arranz de Andrés, 2018), exceptuando de la obligación de pago a los contribuyentes que de algún modo pudieran justificar que el cese de la actividad no oculta ninguna intención fraudulenta en el cobro de la prestación de desempleo en forma de pago único y correspondiente disfrute de la exención fiscal, teniendo el mismo su origen en circunstancias endógenas, vinculadas a la mala gestión del negocio, o exógenas, como las características del mercado o el entorno.

De hecho, en Euskadi el legislador fiscal se ha hecho eco de estas situaciones no queridas o no perseguidas por la persona en situación de desempleo. Así, el plazo de mantenimiento de cinco años de la acción o participación no será exigible cuando su incumplimiento derive de la liquidación de la empresa con motivo de un procedimiento concursal.

Asimismo, estarán exentas las ayudas económicas al empleo por reubicación definitiva reconocidas por las entidades de previsión social voluntaria a las personas socias trabajadoras y de trabajo de cooperativas declaradas disueltas, adscritas al régimen especial de la Seguridad Social de los trabajadores por cuenta propia o autónomos, destinadas a la realización de aportaciones al capital social de las cooperativas en que se reubiquen con carácter definitivo. En estos casos, la cuantía exenta tendrá como límite el importe de la prestación máxima que reconozca por tal concepto el Servicio Público de Empleo Estatal. El exceso tributará como rendimiento del trabajo. Se observa aquí la sensibilidad del legislador fiscal vasco ante el cierre traumático de algunas cooperativas de gran raigambre en Euskadi como el reciente caso de Fagor, que provocó la reubicación de socios en otras cooperativas del grupo.

\subsection{La inversión en microempresas, pequeñas o medianas empresas de nueva o reciente creación o innovadoras}

Para fomentar la financiación del inicio de la actividad económica, existe una deducción en la cuota del 30\% de las cantidades empleadas en la compra de acciones o participaciones de entidades de nueva o reciente creación, que va acompañada de una exención en la transmisión de las acciones o participaciones adquiridas si la desinversión se produce en un determinado plazo. Es un incentivo que beneficia a la persona física contribuyente del IRPF ${ }^{11}$ (business angel) y no directamente a la entidad emprendedora, aunque es ésta quien se beneficia de la financiación por la compra de las acciones o participaciones (Varona Alabern, 2018).

Sin embargo, en la LIRPF se excluye de la deducción y de la exención la inversión en cooperativas puesto que no se admite la inversión en cualquier entidad con personalidad jurídica, sino que debe tratarse de una sociedad anónima, una sociedad de responsabilidad limitada, una sociedad anónima laboral o una sociedad de responsabilidad limitada laboral. Quedan fuera otras formas o clases de sociedad como las cooperativas (Atxabal Rada, 2018c). No se ve cuál es el motivo para excluirlas teniendo en cuenta que pueden ser el cauce adecuado para articular el inicio de pequeñas pero auténticas actividades económicas. Este restrictivo planteamiento ignora la realidad del emprendimiento (Varona Alabern, 2018), que tantas veces se caracteriza por el inicio de una actividad económica de escasa envergadura, para cuyo desarrollo resulta más adecuado adoptar formas jurídicas relativamente simples. Tampoco se encuentra una justificación razonable a la exclusión de las aportaciones realizadas por los socios colaboradores a las cooperativas, a no ser que se trate de las limitaciones en el derecho de separación, una vez cumplido el periodo máximo de inversión que prevé la deducción (Montesinos Oltra, 2017).

Es más, muchas Comunidades Autónomas ${ }^{12}$ han introducido una deducción análoga en la cuota tributaria autonómica para incentivar esta clase de inversiones (Varona Alabern, 2018). Establecen una deducción de

11 El artículo 68.1 de la Ley del IRPF no menciona de manera expresa la figura del business angel, pero sí lo hace tácitamente al indicar que la adquisición de los títulos puede ir acompañada de un asesoramiento por parte del inversor, que podrá aportar conocimientos empresariales o profesionales encaminados a mejorar la actividad económica que desarrolle la empresa de nueva creación (Varona Alabern, 2018).

12 Las Comunidades son Andalucía (art. 15.1 del Decreto Legislativo 1/2018, de 19 de junio), Galicia (art. 5 del Decreto Legislativo 1/2011, de 28 de julio), Aragón (art. 110-9 del Decreto Legislativo 1/2005, de 26 de septiembre), Cataluña (art. 20 de la Ley 26/2009, de 23 de diciembre), las Illes Balears (art. 7 del Decreto Legislativo 1/2014, de 6 de junio), Cantabria (art. 2.6 del Decreto Legislativo 62/2008, de 19 de junio), la Región de Murcia (art. 1.Seis del Decreto Legislativo 1/2010, de 5 de noviembre), Castilla-La Mancha (art. 12 de la Ley 8/2013, de 21 de noviembre), 
entre el $15 \%$ y el $30 \%$ de la cantidad invertida, con un límite máximo de deducción que se sitúa entre un mínimo de 4.000 euros y un máximo de 10.000 euros, dependiendo de cada Comunidad Autónoma. Estas deducciones autonómicas son incompatibles con la deducción estatal de la Ley del IRPF. Y en seis de ellas ${ }^{13}$ se amplía la naturaleza de las entidades destinatarias de la inversión a las sociedades cooperativas.

Al igual que esas comunidades autónomas, en los Territorios Históricos de Álava y Bizkaia, no así en Gipuzkoa, se permite la inversión en cooperativas. Ambos territorios realizan una apuesta por actualizar el incentivo previsto desde 2014 para la inversión en empresas de nueva o reciente creación, mejorando la deducción prevista (Alonso Arce, 2017), incorporando además una deducción más intensa para inversiones de riesgo en microempresas o pequeñas empresas innovadoras, aunque no sean de reciente creación, y complementándola con una exención parcial o total en la enajenación de las participaciones o acciones adquiridas, cuando la desinversión se produzca entre el año 6 y el año 15 de tenencia de la participación, e incorporando una exención en el Impuesto sobre el Patrimonio para esas acciones o participaciones durante los primeros quince años de tenencia de las mismas.

La inversión realizada por el contribuyente puede dar lugar a dos beneficios fiscales distintos: una deducción sobre la base de la inversión realizada y una exención por la enajenación de las participaciones adquiridas con la inversión realizada. Veamos su regulación.

Así, los contribuyentes podrán deducirse el 10\% de las cantidades satisfechas en el período de que se trate por la suscripción de acciones o participaciones en empresas de nueva o reciente creación, pudiendo, además de la aportación temporal al capital, aportar sus conocimientos empresariales o profesionales adecuados para el desarrollo de la entidad en la que invierten, en los términos que establezca el acuerdo de inversión entre el o la contribuyente y la entidad. Hablamos del inversor de proximidad o business angel.

La base máxima de deducción será de 100.000 euros anuales y estará formada por el valor de adquisición de las acciones o participaciones suscritas. Asimismo, la cantidad máxima anual deducible no podrá superar el 15\% de la base liquidable de este Impuesto correspondiente al contribuyente. Las cantidades no deducidas por superarse los anteriores límites, podrán aplicarse, respetando los mismos, en las autoliquidaciones de los periodos impositivos que concluyan en los cinco años inmediatos y sucesivos.

La misma deducción será del $20 \%$ de las cantidades satisfechas en el período de que se trate cuando las acciones o participaciones suscritas correspondan a microempresas, pequeñas o medianas empresas innovadoras ${ }^{14}$. En estos casos, la base máxima de deducción será de 150.000 euros anuales y la cantidad máxima anual deducible no podrá superar el $15 \%$ de la base liquidable de este Impuesto correspondiente al contribuyente.

Las acciones o participaciones en la entidad deberán adquirirse por el o por la contribuyente bien en el momento de la constitución de aquélla o mediante ampliación de capital efectuada en los cinco años siguientes a dicha constitución y permanecer en su patrimonio por un plazo superior a cinco años.

En el supuesto de invertir en microempresas, pequeñas o medianas empresas innovadoras, las acciones o participaciones deberán adquirirse en el plazo de los siete años siguientes a dicha constitución, aunque este plazo no será exigible en el supuesto de entidades que necesiten una inversión inicial de financiación de riesgo que, sobre la base de un plan de negocio elaborado con vistas a introducirse en un nuevo mercado geográfico o de productos, sea superior al $50 \%$ de la media de su volumen de operaciones anual en los cinco años anteriores.

Junto a la deducción que acabamos de comentar, se regula una exención relacionada con la transmisión de las acciones. Estarán exentas del Impuesto las ganancias patrimoniales que se pongan de manifiesto con ocasión de la transmisión de las acciones o participaciones en entidades por cuya adquisición el contribuyente hubiera aplicado la deducción por la inversión en microempresas, pequeñas o medianas empresas de nueva o reciente creación o innovadoras, si la transmisión de las mismas se produce entre el sexto y el décimo año desde la adquisición. Esta opción tampoco se regula en Gipuzkoa.

La aplicación de esta medida presupone la existencia de una ganancia patrimonial derivada de la transmisión de las acciones o participaciones. El legislador no ha previsto ningún beneficio fiscal en caso de producirse una pérdida patrimonial, posiblemente por entender que, si no hay riqueza gravable, tampoco nace una obligación tributaria digna de ser reducida (Varona Alabern, 2018).

La exención alcanzará al 50\% de la ganancia patrimonial cuando la transmisión de las acciones o participaciones se produzca entre el undécimo y el decimoquinto año. No hay exención si la transmisión se produce en los primeros cinco años o una vez transcurridos quince desde la adquisición.

Extremadura (art. 11 del Decreto Legislativo 1/2018, de 10 de abril) y las Comunidades de Madrid (art. 15 del Decreto Legislativo 1/2010, de 21 de octubre) y Castilla y León (art. 8 del Decreto Legislativo 1/2013, de 12 de septiembre).

13 Así sucede en Andalucía, Galicia, Murcia, Castilla-La Mancha (para las cooperativas agropecuarias), Extremadura y la Comunidad de Madrid

14 A estos efectos, se considerarán empresas innovadoras las que cumplan, en el ejercicio de toma de la participación, lo dispuesto en el apartado 80 del artículo 2 del Reglamento (UE) número 651/2014, de la Comisión, de 17 de junio de 2014, por el que se declaran determinadas categorías de ayudas compatibles con el mercado interior en aplicación de los artículos 107 y 108 del Tratado. 


\subsection{La deducción por inversión en Fondos Europeos para el impulso de la innovación}

Tanto Álava como Bizkaia regulan el tratamiento tributario de determinados fondos europeos. A modo de resumen general el esquema de los fondos tratados en estos territorios forales es el siguiente:

- - Fondos de inversión a largo plazo europeos:

- - Para el impulso de la innovación.

- - Para el impulso de la financiación de la actividad económica.

- - Para el impulso de la capitalización productiva.

- - Fondos de capital riesgo europeos.

- - Fondos de emprendimiento social europeos.

Estos fondos, con la autorización de las Administraciones públicas forales, posibilitan la intermediación entre los particulares y los empresarios que llevan a cabo actividades innovadoras y emprendedoras, que serán financiadas por los fondos así constituidos (Atxabal Rada, 2018c).

A efectos del Impuesto sobre Sociedades, los Fondos de capital riesgo europeos (FCRE) y los Fondos de emprendimiento social europeo (FESE) tendrán el régimen tributario de los fondos de capital riesgo, consistente en la exención de los dividendos y las plusvalías de las participaciones, cualesquiera que sea el porcentaje de participación y la antigüedad de la misma. En el caso de las plusvalías, siempre que no hayan pasado 15 años desde la adquisición de la participación, podrán acogerse a este beneficio fiscal las rentas derivadas de valores que tengan la consideración de inversiones admisibles. Por su parte, los Fondos de inversión a largo plazo europeos (FILPE) que cumplan los requisitos establecidos tributarán en el Impuesto sobre Sociedades al tipo del $1 \%$.

Se trata de una fórmula indirecta de inversión colectiva destinada a la concesión de préstamos a empresas innovadoras o emprendedoras entre las que se pueden encontrar las cooperativas. No es, por tanto, un incentivo dirigido expresamente a las cooperativas pero éstas se pueden beneficiar del mismo al igual que las demás clases de empresa.

Los contribuyentes podrán aplicar una deducción del $15 \%$ de las cantidades satisfechas en el período impositivo, destinadas a la adquisición de participaciones de Fondos europeos para el impulso a la innovación que cumplan los requisitos previstos en la normativa foral. Asimismo, los contribuyentes podrán aplicar idéntica deducción por las cantidades que se depositen en entidades de crédito, en cuentas destinadas a la adquisición de participaciones en dichos fondos. La deducción no podrá superar la cantidad de 750 euros anuales, por lo que la inversión de cada partícipe con derecho a deducción no podrá superar los 5.000 euros.

\section{Otros incentivos fiscales para el emprendimiento que excluyen a las cooperativas}

No terminan ahí las medidas que impulsan el emprendimiento. Las normativas del IRPF y del Impuesto sobre Sociedades recogen otras medidas dirigidas a favorecer el emprendimiento, aunque en estos casos las cooperativas no pueden ser beneficiarias de los mismos, bien porque las excluyan directamente bien porque no pueden cumplir los requisitos previstos para la aplicación del incentivo que se basan en las sociedades de capital e ignoran las características propias de las cooperativas.

\subsection{Las deducciones por participación de los trabajadores en la empresa}

Además de la exención hasta 12.000 euros de la renta en especie por adquisición de acciones de los trabajadores prevista en el artículo 42.2.f) de la LIRPF, y por tanto compatible con la misma, en Euskadi se regulan dos deducciones relacionadas con la participación de los trabajadores en la empresa. Por un lado, se regula la adquisición de acciones o participaciones de la entidad para la que trabajan o de cualquiera otra del grupo de sociedades al que pertenece la entidad para la que prestan sus servicios. Por otro lado, se regula una deducción también para la adquisición de acciones o participaciones de su entidad o cualquiera del grupo, inversión que se haya financiado mediante préstamos de fondos públicos del Gobierno vasco.

Los contribuyentes podrán aplicar una deducción del 10\%, con un límite anual máximo de 1.200 euros, de las cantidades satisfechas en metálico en el período impositivo, destinadas a la adquisición o suscripción de acciones o participaciones en la entidad o en cualquiera del grupo de sociedades, en la que prestan sus servicios como trabajadores.

Asimismo, los contribuyentes podrán aplicar una deducción del $10 \%$, con un límite anual máximo de 6.000 euros, de las cantidades satisfechas en metálico en el período impositivo, provenientes de préstamos de Fondos constituidos por las Administraciones Públicas vascas, que se destinen a la adquisición o suscripción de acciones o participaciones en la entidad o en cualquiera del grupo de sociedades, en la que prestan sus servicios como trabajadores. 
Es cierto que las cooperativas no están expresamente excluidas de las deducciones. Sin embargo, no se menciona la adquisición de participaciones por los socios de la cooperativa sino que el incentivo se dirige a los trabajadores por cuenta ajena para que participen en el capital de la empresa donde trabajan por lo que su aplicabilidad a las cooperativas se restringirá a aquellos trabajadores de la misma que adquieran sus participaciones en el capital cuando se transformen en socios de la misma, si bien a partir de ese momento dejarán de ser trabajadores por lo que se podría cuestionar la aplicación de la deducción también en ese supuesto. La exclusión de las cooperativas se puede explicar porque la propia naturaleza de la cooperativa garantiza la finalidad perseguida por el incentivo, esto es, la participación de los trabajadores en su empresa.

\subsection{Deducción por la constitución de entidades por las personas trabajadoras}

En Gipuzkoa, se regula una deducción del 10\% si son hombres, o del 15\% si son mujeres, de las cantidades satisfechas en metálico en el período impositivo, destinadas a la suscripción de acciones o participaciones para la constitución de la entidad en la que prestarán sus servicios como personas trabajadoras.

Las acciones o participaciones que dan derecho a esta deducción deberán mantenerse durante al menos los cinco años siguientes a la constitución. El contribuyente deberá prestar sus servicios como trabajador de la entidad como mínimo durante el citado periodo, salvo que, antes de cumplir dicho plazo, falleciera o hubiera dejado de trabajar en la misma como consecuencia de despido o de que le hubiera sido reconocida una situación de incapacidad permanente total o absoluta o de gran invalidez.

Las cooperativas quedan fuera de esta deducción por razones similares a las expuestas en el apartado precedente.

\subsection{La deducción por financiación a entidades con alto potencial de crecimiento}

Por su parte, en el Territorio Histórico de Gipuzkoa (Alonso Arce, 2017), incorporan una nueva deducción para determinadas inversiones de riesgo en determinados sectores que se eleva al $30 \%$ de las inversiones con una base máxima de deducción de 1.000 .000 euros, incorporando una medida sin parangón en el resto de normativas del IRPF.

Se articulan dos modalidades de deducción:

a) Una deducción del $30 \%$ de las cantidades satisfechas en metálico en el periodo impositivo por la suscripción, en el mercado primario, de acciones y participaciones en entidades innovadoras de nueva creación.

b) Una deducción del $15 \%$ de las cantidades satisfechas en metálico en el período impositivo por la suscripción, en el mercado primario, de acciones o participaciones en entidades en proceso de crecimiento.

Sin embargo, las cooperativas quedan fuera de este incentivo fiscal puesto que, para aplicar la deducción, se exige que la entidad cuyas acciones o participaciones se suscriban revista la forma de sociedad anónima, sociedad de responsabilidad limitada, sociedad anónima laboral o sociedad de responsabilidad limitada laboral.

\subsection{La reserva especial para el fomento del emprendimiento y el reforzamiento de la actividad productiva}

Los contribuyentes podrán reducir su base imponible en el 60\% del importe de su resultado contable positivo que destinen a la dotación de una reserva especial para el fomento del emprendimiento y el reforzamiento de la actividad productiva. En este caso no va a ser el total importe que se aplique a la reserva el que dé lugar a una corrección del resultado contable para determinar la base imponible, sino el $60 \%$ de ese importe. La razón de ser de esta limitación es conceder un incentivo del 14,4\% (el 60\% del tipo general de gravamen, o del $12 \%$, si estamos hablando de empresas que tributan en el Impuesto sobre Sociedades como microempresas o pequeñas empresas al 20\%), ya que se quiere que guarde relación cuantitativa directa con otros incentivos fiscales a los que sustituye (Alonso Arce, 2014).

Las cantidades dotadas a la reserva especial para el fomento del emprendimiento y el reforzamiento de la actividad productiva deberán destinarse, en el plazo máximo de tres años desde el último día del período impositivo con cargo a cuyo resultado contable positivo se hubiera dotado la mencionada reserva especial, a la adquisición de activos no corrientes nuevos, a la adquisición de elementos patrimoniales con una finalidad medioambiental, a la adquisición en el mercado primario de participaciones en entidades como vía de fomento directo del emprendimiento, del desarrollo de nuevos proyectos y de otorgar financiación a las nuevas líneas empresariales desde una perspectiva de reinvertir los beneficios de la actividad en crear nuevos productos, mercados o proyectos (Atxabal Rada, 2018c), o a la inversión mediante la participación en el 
capital en la etapa inicial de desarrollo de un nuevo proyecto empresarial o en su fase de desarrollo, de las microempresas y pequeñas y medianas empresas con alto potencial de crecimiento.

No podrán aplicar, en ningún caso, esta reserva las entidades sometidas al Impuesto, salvo las que tributen al tipo general del Impuesto, las microempresas y pequeñas empresas, y las entidades de crédito aunque formen parte de un grupo de empresas. También se excluyen las entidades que estén sometidas a los regímenes especiales establecidos en el Impuesto sobre Sociedades, salvo los grupos fiscales que tributen en el régimen especial de consolidación fiscal. Las cooperativas quedan excluidas por dos vías: porque no aplican el tipo general del impuesto y porque tienen un régimen fiscal especial en el Impuesto sobre Sociedades.

\section{Conclusiones}

La cooperativa puede impulsar el emprendimiento, al igual que otras formas de empresa. De hecho, el inicio de una actividad económica no está limitado a una clase de empresa en particular, sino que es posible emplear la fórmula que mejor se adapte a las necesidades de los emprendedores, entre las que, sin duda, podemos mencionar a las cooperativas (Arnáez Arce, 2018a). Esta realidad, sin embargo, no tiene un reflejo equivalente en la normativa fiscal española.

Las normas tributarias que fomentan el emprendimiento, adoptadas desde 2013 hasta la actualidad, no recogen medidas específicas para las cooperativas, ni para las entidades de economía social (Montesinos Oltra, 2017). Encontramos, a lo sumo, menciones a alguna forma de empresa de la economía social, principalmente a las sociedades laborales y en mucha menor medida a las cooperativas, como acreedoras de la aplicación de las medidas incentivadoras del emprendimiento junto a otras formas de empresa, lo que supone englobar a las cooperativas (cuando lo hace el legislador) dentro de las medidas fiscales generales.

Observaremos que algunas medidas fiscales que impulsan el emprendimiento podrán ser aplicadas también por las cooperativas; y sin embargo, la utilización de otras medidas fiscales se limitará a determinadas formas de empresa, siempre las empresas capitalistas, entre las que no se incluyen las cooperativas. No existe una justificación para esta diferencia de trato en la normativa fiscal. Se pone en cuestión el principio de igualdad tributaria que debe primar en la normativa fiscal, ex artículo 31 de la Constitución.

Las cooperativas pueden acogerse a alguno de los incentivos fiscales al emprendimiento como un menor tipo de gravamen si son entidades de nueva creación, una laxitud en la aplicación de los límites conjuntos a las deducciones del Impuesto sobre Sociedades, una deducción para la creación de empleo mediante el contrato de trabajo de apoyo al emprendimiento, la exención de la prestación por desempleo si se cobra en forma de capital, y en el caso de Euskadi, la deducción por inversión en empresas de nueva o reciente creación y una deducción por la inversión en fondos europeos.

Sin embargo, se excluye a las cooperativas de otros incentivos como la regla más favorable de la compensación de bases imponibles negativas, la deducción por inversión en entidades de nueva creación, o en el caso de Euskadi, varias deducciones aplicables en el IRPF y en el IS.

Coincidimos con Montesinos Oltra (2017: 14) cuando afirma que "el legislador tributario no sólo no muestra una especial sensibilidad hacia la economía social en la regulación de las medidas de incentivo al emprendimiento adoptadas en los últimos tiempos, sino que, más bien al contrario, se olvida de ellas o incluso las discrimina en su aplicación por el simple hecho de que les resulte aplicable en el Impuesto sobre Sociedades, en su caso, un tipo de gravamen inferior al general. Y esto, si bien podría haber servido en el pasado de justificación o, al menos, de explicación razonable, pierde sentido en la actualidad si tenemos en cuenta que la nueva LIS ha supuesto un paso más en la aproximación de las entidades de la economía social que cuentan con algún régimen especial al tratamiento que reciben los contribuyentes en régimen general en cuanto al tipo de gravamen aplicable".

No se vislumbran los motivos que han llevado al legislador fiscal, sea de territorio común o de territorio foral, a eliminar a las cooperativas del grupo de beneficiarios de estos incentivos al emprendimiento. Es más, parece que la finalidad perseguida con estas medidas fiscales de impulsar el emprendimiento y facilitar la creación de empleo se puede conseguir a través de sociedades capitalistas y mediante sociedades cooperativas. Entonces, ¿por qué hace estas diferencias el legislador fiscal?

Podríamos buscar una explicación en el hecho de que los entes de la economía social no sean especialmente proclives al emprendimiento para el legislador fiscal. Pero, incluso si así fuera (y habría que demostrarlo), resulta más paradójico el hecho de que las sociedades laborales sí se puedan acoger a estos beneficios fiscales, mientras que las cooperativas quedan excluidas. Aunque ambas sean entes que forman parte de la economía social, el legislador fiscal hace distingos entre ellas.

Es cierto que la regulación fiscal de las entidades de economía social, y de las cooperativas en particular, parten de una dispersión normativa (Rodrigo Ruiz, 2017) donde existen previsiones fiscales singulares o particulares para las cooperativas junto a textos legales generales que regulan los principales impuestos y que 
a su vez resultan de aplicación a las cooperativas. Ello genera una dificultad añadida por la dispersión temporal o cronológica que supone, ya que la norma especial y la general no se modifican al unísono lo que provoca la obsolescencia de la primera, que no es objeto de reforma, mientras que la normativa general se va adecuando a los tiempos (Rodrigo Ruiz, 2017). Por ejemplo, la normativa tributaria especial de las cooperativas es un texto desfasado y obsoleto, hoy superado por la legislación emanada con posterioridad, tanto sustantiva como tributaria general.

Sea como fuere, las cooperativas, en cuanto que emprendan una actividad económica, son acreedoras de la aplicación de algunos incentivos fiscales. Es cierto que no hay incentivos fiscales específicos para el emprendimiento impulsado por las cooperativas, pero no es menos cierto, que éstas pueden acogerse a algunos incentivos fiscales que fomentan el emprendimiento, al igual que cualquier otra empresa emprendedora.

\section{Referencias bibliográfícas}

AAVV (2018) Memento Práctico Fiscal 2018. Madrid: Francis Lefebvre.

Alonso Arce, I. (2017) La reforma fiscal de los Territorios Históricos vascos para 2018. Forum Fiscal, la revista tributaria de Álava, Bizkaia y Gipuzkoa, № 237.

Alonso Arce, I. (2014) Las nuevas reservas en el Impuesto sobre Sociedades: las correcciones en materia de aplicación del resultado. Forum fiscal: la revista tributaria de Álava, Bizkaia y Gipuzkoa, № 196, pp. 19-44.

Alonso Rodrigo, E. (2001) Fiscalidad de cooperativas y sociedades laborales. Barcelona: Institut per a la Promoció y la Formació Cooperatives, Generalitat de Catalunya.

Armentia Basterra, J. (2014) Régimen fiscal aplicable a las microempresas, pequeñas y medianas empresas en la nueva normativa del Impuesto sobre Sociedades. Zergak: gaceta tributaria del País Vasco, № 48, pp. 91-106.

Arnáez Arce, V.M. (2018a) La alternativa cooperativa en la prestación de servicios públicos sostenibles. Boletín de la Asociación Internacional de Derecho Cooperativo, No 52, pp. 123-135, doi: http://dx.doi.org/10.18543/baidc-522018pp123-135.

Arnáez Arce, V.M. (2018b) El modelo cooperativo como alternativa sostenible para la gestión de los servicios públicos. En: Arnáez Arce, V.M. e Izquierdo Muciño, M.E. (coords.) Fomento del Cooperativismo como alternativa económica y social sostenible: una visión de México y España. Madrid: Dykinson, pp. 109-120.

Arranz de Andrés, C. (2018) El trabajador por cuenta propia emprendedor en el impuesto sobre la renta de las personas físicas. En Varona Alabern (dir.) La Fiscalidad del Emprendimiento. Cizur Menor (Navarra): Thomson-Reuters Aranzadi, pp. 99-156.

Arrázola Arrién, F. (2015) Diferencias más importantes de la normativa foral y de territorio común, una vez aprobada la Ley 27/2014 del Impuesto sobre Sociedades. Forum fiscal: la revista tributaria de Álava, Bizkaia y Gipuzkoa, № 208, pp. 96-100.

Atxabal Rada, A. (2018a) Los impuestos personales sobre el capital en las cooperativas y en sus socios en el País Vasco. Boletín de la Asociación Internacional de Derecho Cooperativo, $\mathrm{N}^{\mathrm{o}}$ 52, pp. 137-166, doi: http://dx.doi.org/10.18543/baidc-52-2018pp137-166.

Atxabal Rada, A. (2018b) El emprendedor societario en el impuesto sobre sociedades. En Varona Alabern (dir.) La Fiscalidad del Emprendimiento. Cizur Menor (Navarra): Thomson-Reuters Aranzadi, pp. 209-252.

Atxabal Rada, A. (2018c) Las principales medidas fiscales para el fomento del emprendimiento en la comunidad autónoma del País Vasco. En Varona Alabern (dir.) La Fiscalidad del Emprendimiento. Cizur Menor (Navarra): Thomson-Reuters Aranzadi, pp. 567-597.

Atxabal Rada, A. (2014) La compensación de bases imponibles negativas. Forum fiscal: la revista tributaria de Álava, Bizkaia y Gipuzkoa, No 199, pp. 39-57.

Belso Martínez, J.A.; Chinchilla Mira, L.; López Sánchez, M.J.; Pérez Martín, A. (2014) La empresa y el emprendimiento. En Ribeiro Soriano, D. (aut.) Creación de empresas y emprendimiento: de estudiante a empresario. Madrid: Pearson Educación, pp. 25-44.

Chaves, R. y Monzón, J.L. (2018) La economía social ante los paradigmas económicos emergentes: innovación social, economía colaborativa, economía circular, responsabilidad social empresarial, economía del bien común, empresa social y economía solidaria. CIRIEC-España, Revista de Economía Pública, Social y Cooperativa, No 93, pp. 5-50, doi: 10.7203/CIRIEC-E.93.12901.

Global Entrepreneurship Monitor. (2018) Informe GEM España 2017-2018. Santander: CISE, Editorial Universidad de Cantabria.

Lagos Rodríguez, M.G. y Álamo Cerrillo, R. (2016) Emprendimiento y fiscalidad. Carta Tributaria. Revista de opinión, $\mathrm{N}^{\mathrm{o}} 15, \mathrm{pp} .37-49$.

Luján Alcaraz, J. (2016) El fomento del emprendimiento. En Luján Alcaraz, J. y Ferrando García, F.M. (dirs.), Trabajo autónomo y fomento del emprendimiento: mitos y realidades. Albacete: Bomarzo, pp. 9-42.

Montero Simó, M. (2016) La fiscalidad de las cooperativas ante el nuevo Impuesto sobre Sociedades. Boletín de la Asociación Internacional de Derecho Cooperativo, $\mathrm{N}^{\circ}$ 50, pp. 17-47. doi: http://dx.doi.org/10.18543/baidc-502016pp17-.47.

Montesinos Oltra, S. (2017) Incentivos fiscales al emprendimiento y economía social. Lex Social, vol. 7, № 2, pp. 4-37. 
Palomo Ruiz, L. (2015) Ley de Emprendedores: un balance de resultados tras dos años de vigencia. Estudios financieros. Revista de trabajo y seguridad social: Comentarios, casos prácticos : recursos humanos, №. 387 , pp. $65-98$.

Pérez Delgado, A. (2014) Las microempresas y las PYMES en las nuevas normas forales del Impuesto sobre Sociedades de los territorios históricos del País Vasco. Forum fiscal: la revista tributaria de Álava, Bizkaia y Gipuzkoa, No 196 , pp. 45-60.

Rodrigo Ruiz, M.A. (2017) Consideraciones en torno al establecimiento de un régimen tributario común para las entidades de la economía social. REVESCO, Revista de Estudios Cooperativos, Tercer Cuatrimestre, $\mathrm{N}^{\circ} 125$, pp. 187-212, doi: http://dx.doi.org/10.5209/REVE.57064.

Romero Flor, L.M. y Álamo Cerrillo, R. (2014a) Análisis de los incentivos fiscales introducidos en el ámbito del Impuesto sobre la Renta de las personas físicas y jurídicas para el fomento del emprendimiento. Revista Jurídica de Castilla-La Mancha, № 55, pp. 139-164.

Romero Flor, L.M. y Álamo Cerrillo, R. (2014b) Incentivos fiscales al emprendimiento. Quincena Fiscal, № 17, pp. 89-107.

Ruiz Garijo, M. (2016) Incentivos al emprendimiento en el Impuesto sobre Sociedades. En: Merino Jara, I. y García Luis, T. (coord.) La reforma del Impuesto sobre Sociedades. Madrid: Instituto de Estudios Fiscales, pp. 609-638.

Vaquero García, A. (2016) Actuaciones fiscales en materia de emprendimiento: resultados y líneas de mejora, Documentos, $\mathrm{N}^{\mathrm{o}} 24$, Instituto de Estudios Fiscales.

Varona Alabern, J.E. (2018) Los "business angels" en el impuesto sobre la renta de las personas físicas. En Varona Alabern (dir.) La Fiscalidad del Emprendimiento. Cizur Menor (Navarra): Thomson-Reuters Aranzadi, pp. 71-97.

Zapata Huamaní, G.A., Fernández López, S. y Neira Gómez, I. (2018) Nivel tecnológico de las iniciativas emprendedoras. Una perspectiva internacional. En: VV.AA., Global Entrepreneurship Monitor. Informe GEM España 2017-2018. Santander: Universidad de Cantabria, pp. 125-137. 